\title{
Quantitative DCE-MRI demonstrates increased blood perfusion in Hoffa's fat pad signal abnormalities in knee osteoarthritis, but not in patellofemoral pain
}

\author{
Bas A. de Vries ${ }^{1} \cdot$ Rianne A. van der Heijden ${ }^{1} \cdot$ Dirk H. J. Poot ${ }^{1,2} \cdot$ Marienke van Middelkoop $^{3} \cdot$ Duncan E. Meuffels $^{4} \cdot$ \\ Gabriel P. Krestin ${ }^{1}$ • Edwin H. G. Oei ${ }^{1}$ (D)
}

Received: 16 October 2019 /Revised: 19 December 2019 / Accepted: 23 January 2020 / Published online: 17 February 2020

(C) The Author(s) 2020

\begin{abstract}
Objective Infrapatellar fat pad (IPFP) fat-suppressed T2 (T2 $\mathrm{FS}$ ) hyperintense regions on MRI are an important imaging feature of knee osteoarthritis (OA) and are thought to represent inflammation. These regions are also common in non-OA subjects, and may not always be linked to inflammation. Our aim was to evaluate quantitative blood perfusion parameters, as surrogate measure of inflammation, within $\mathrm{T} 2{ }_{\mathrm{FS}}$-hyperintense regions in patients with OA, with patellofemoral pain (PFP) (supposed OA precursor), and control subjects.

Methods Twenty-two knee OA patients, 35 PFP patients and 43 healthy controls were included and underwent MRI, comprising T2 and DCE-MRI sequences. T2 ${ }_{\mathrm{FS}}$-hyperintense IPFP regions were delineated and a reference region was drawn in adjacent IPFP tissue with normal signal intensity. After fitting the extended Tofts pharmacokinetic model, quantitative DCE-MRI perfusion parameters were compared between the two regions within subjects in each subgroup, using a paired Wilcoxon signed-rank test.

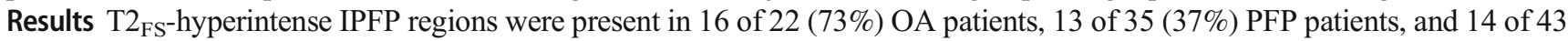
$(33 \%)$ controls. DCE-MRI perfusion parameters were significantly different between regions with and without a T2 ${ }_{\mathrm{FS}}-\mathrm{hyperin}-$ tense signal in OA patients, demonstrating higher Ktrans compared to normal IFPF tissue $\left(0.039 \mathrm{~min}^{-1}\right.$ versus $0.025 \mathrm{~min}^{-1}, p=$ $0.017)$ and higher Ve $(0.157$ versus $0.119, p=0.010)$. For PFP patients and controls no significant differences were found.

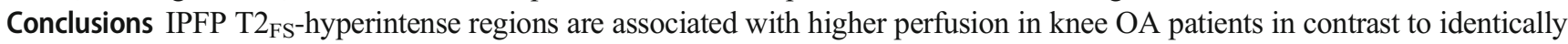
appearing regions in PFP patients and controls, pointing towards an inflammatory pathogenesis in OA only.

Key Points

- Morphologically identical appearing $T 2_{F S}$-hyperintense infrapatellar fat pad regions show different perfusion in healthy subjects, subjects with patellofemoral pain, and subjects with knee osteoarthritis.

- Elevated DCE-MRI perfusion parameters within $T 2_{F S}$-hyperintense infrapatellar fat pad regions in patients with osteoarthritis suggest an inflammatory pathogenesis in osteoarthritis, but not in patellofemoral pain and healthy subjects.
\end{abstract}

Keywords Osteoarthritis, knee $\cdot$ Patellofemoral pain syndrome $\cdot$ Inflammation $\cdot$ Magnetic resonance imaging $\cdot$ Synovitis

Bas A. de Vries and Rianne A. van der Heijden shared first authorship

Edwin H. G. Oei

e.oei@erasmusmc.nl

1 Department of Radiology \& Nuclear Medicine, Erasmus University Medical Center Rotterdam, Rotterdam, The Netherlands

2 Department of Medical Informatics, Erasmus University Medical Center Rotterdam, Rotterdam, The Netherlands

3 Department of General Practice, Erasmus University Medical Center Rotterdam, Rotterdam, The Netherlands

4 Department of Orthopedic Surgery, Erasmus University Medical Center Rotterdam, Rotterdam, The Netherlands

$\begin{array}{ll}\text { Abbreviations } \\ \text { AIF } & \text { Arterial input function } \\ \text { BMI } & \text { Body mass index } \\ \text { DCE-MRI } & \text { Dynamic contrast-enhanced MRI } \\ \text { FOV } & \text { Field-of-view } \\ \text { FSE } & \text { Fast spin echo } \\ \text { FSPGR } & \text { Fast spoiled gradient echo } \\ \text { IPFP } & \text { Infrapatellar fat pad or Hoffa's fat pad } \\ \text { IQR } & \text { Interquartile range } \\ \text { KL } & \text { Kellgren and Lawrence } \\ \text { KOOS } & \text { Knee Injury and Osteoarthritis Outcome Score } \\ \text { MOAKS } & \text { MRI Osteoarthritis Knee Score }\end{array}$




$\begin{array}{ll}\text { MRI } & \text { Magnetic resonance imaging } \\ \text { OA } & \text { Osteoarthritis } \\ \text { PFP } & \text { Patellofemoral pain } \\ \text { ROI } & \text { Region of Interest } \\ \text { SD } & \text { Standard deviation }\end{array}$

\section{Introduction}

The infrapatellar fat pad (IPFP), also known as "Hoffa's fat pad," is an intracapsular, extra-synovial structure in the anterior knee joint and is one of several fat pads of the knee. This structure has been proposed as possible source of knee pain in patients suffering from osteoarthritis (OA) and from the supposed precursor of knee OA: patellofemoral pain (PFP) [1-6]. In OA research, the MRI Osteoarthritis Knee Score (MOAKS) is one of the most commonly used scoring systems for knee OA on MRI [7]. In this method, the presence and size of hyperintense signal within the IPFP is scored on unenhanced fat-suppressed MR images. These hyperintense lesions are thought to be a manifestation of knee inflammation and are therefore classified as Hoffa synovitis [7]. Moreover, multiple studies have emphasized the importance of $\mathrm{T}_{\mathrm{FS}}-$ hyperintense IPFP regions as a precursor for structural knee OA [8-12].

A recent study that included patients with PFP and healthy controls subjects showed that $\mathrm{T} 2 \mathrm{FS}_{\mathrm{FS}}$-hyperintense regions in the IPFP are rather common [13]. The question arises whether this identically appearing feature, commonly encountered in daily clinical practice and considered an "early OA" feature, has a different pathophysiology across populations. A hyperintense $\mathrm{T} 2_{\mathrm{FS}}$ signal may be caused by edema due to inflammatory induced vasodilatation, but a prior study by Roemer et al suggested that this feature may not always be linked to inflammation [14]. Other causative effects of a fluid signal might be edema due to mechanical friction/impingement or increased vascularity due to neo-angiogenesis, necrosis, or cellular infiltration $[15,16]$.

Dynamic contrast-enhanced (DCE) MRI enables further evaluation of the pathophysiology of IPFP $\mathrm{T} 2{ }_{\mathrm{FS}}$-hyperintense lesions. Fitting a pharmacokinetic model to the DCE-MRI data enables quantitative surrogate measurement of physiological parameters such as blood flow, blood volume, and extravascular permeability [17]. Increased blood perfusion, evaluated by DCE-MRI, has been considered a surrogate measure of inflammation for a variety of musculoskeletal tissues [18-23]. To the best of our knowledge, the only research with regard to DCE-MRI in the IPFP was performed by Ballegaard et al [23]. They studied obese patients with knee OA using a heuristic DCE-MRI analysis approach and found a positive correlation between knee pain and their DCE-MRI-derived inflammation marker and between knee pain and Hoffasynovitis assessed by MOAKS, thereby stipulating the importance of the IPFP and the potential of DCE-MRI as a biomarker of inflammation [23]. So far, DCE-MRI has not been applied for studying the pathogenesis of $\mathrm{T} 2_{\mathrm{FS}}$-hyperintense IPFP regions in an OA and non-OA population.

Therefore, the aim of this study was to evaluate differences in quantitative DCE-MRI blood perfusion parameters between a $\mathrm{T} 2 \mathrm{FS}_{\mathrm{FS}}$-hyperintense region of the IPFP and adjacent IPFP tissue with normal signal intensity within patients with knee OA, patients with PFP and healthy control subjects. Our hypothesis was that $\mathrm{T} 2{ }_{\mathrm{FS}}$-hyperintense IPFP regions demonstrate different DCE-MRI perfusion parameters in patients with OA, patients with PFP, and healthy control subjects, with the highest degree of perfusion expected in patients with OA.

\section{Materials and methods}

\section{Study population}

In the current study, we analyzed data from two previous studies in order to include both patients with OA and patients with PFP, the supposed precursor of OA. In the first study, patients with unicompartmental radiographic knee OA with a severity of KL (Kellgren and Lawrence [24]) grade 2 and higher, aged 52 to 75 years, scheduled to undergo knee replacement surgery, were included. Patients were excluded when the glomerular filtration rate was $<60 \mathrm{~mL} / \mathrm{min}$. In the second study, healthy controls and patients with PFP, aged between 18 and 40 years, were included. Patients were excluded if they had other defined pathological conditions of the knee such as patellar tendinopathy or osteoarthritis, if the onset of PFP occurred after trauma, if they had previous knee injuries or surgery or previous episodes of PFP that occurred more than 2 years ago, or if they had contraindications for MRI scanning with contrast administration. Patients of both studies were included between 2013 and 2017 at the Erasmus University Medical Center Rotterdam (Rotterdam, The Netherlands); details of each study have been published elsewhere $[13,25]$. Both studies were approved by the institutional review board of Erasmus MC and written informed consent was obtained from all subjects.

\section{MR imaging acquisition}

In both studies, all subjects underwent MRI using the same MRI scanner and an identical MRI protocol. Multisequence MRI was performed using a 3-T MRI system (Discovery MR750, General Electric Healthcare) and a dedicated 8channel transmit/receive knee coil (Invivo). DCE-MRI was acquired in the sagittal plane, using a fat-suppressed 3D fast spoiled gradient echo (FSPGR) sequence with 35 phases of $10 \mathrm{~s}$. Contrast agent $0.2 \mathrm{mmol} / \mathrm{kg}$ gadopentetate dimeglumine (Magnevist, Bayer) was administrated intravenously with a 
power injector at a rate of $2 \mathrm{ml} / \mathrm{s}$ started after the first phase and followed by a saline flush. The field-of-view (FOV) was $22 \times 22 \mathrm{~cm}$, with an in-plane resolution of $0.85 \times 1.20 \mathrm{~mm}$ and 5 -mm slice thickness, and a flip angle of $30^{\circ}$ and repetition time of $9.3 \mathrm{~ms}$ was used. T2 mapping was performed using a iMSDE prepared 3D fast spin echo (FSE) sequence with a FOV of $15 \times 15 \mathrm{~cm}, 3-\mathrm{mm}$ slice thickness, and an in-plane resolution of $0.52 \times 0.78 \mathrm{~mm}$, using 5 different echo times in the preparation module $(3.1,13.4,27.0,40.7,68.1 \mathrm{~ms})$. The protocol also included a fat-suppressed sagittal T2-weighted FSE sequence with a FOV of $15 \times 15 \mathrm{~cm}, 3$-mm slice thickness, and an in-plane resolution of $0.36 \times 0.59 \mathrm{~mm}$.

\section{Image analysis}

To correct for patient movement, all 35 time points of the DCE-MRI were registered using an automated rigid body registration with Elastix [26]. We first assessed the fat-saturated $\mathrm{T} 2$-weighted images for the presence of $\mathrm{T} 2 \mathrm{FS}_{\mathrm{FS}}$-hyperintense regions in the IPFP. Subsequently, detected $\mathrm{T} 2_{\mathrm{FS}}$-hyperintense regions were delineated on the quantitative T2 maps. The delineation of ROIs was performed on T2 maps as these images were scanned in the same part of the scan session as the DCE-MRI, in contrast to the $\mathrm{T} 2 \mathrm{FS}$-weighted images, and thus the regions of interest (ROIs) could be copied to the DCEmaps. ROIs were placed within the borders of the hyperintense regions using the Horos software package (Horosproject.org). When multiple hyperintense regions were found in the IPFP, the ROI was placed in only one, the largest region. Two ROIs were drawn in the IPFP, one within the $\mathrm{T} 2{ }_{\mathrm{FS}}$-hyperintense region and the second in an adjacent area without T2-hyperintensity (Fig. 1). All ROIs were drawn by a researcher with a technical medical degree and more than three years of experience in musculoskeletal imaging research (B.d.V.). The same ROIs from the T2-maps were copied to the registered DCE-MR images to extract quantitative DCE measures from the same regions. These quantitative DCE parameters (Ktrans, Kep, Ve, Vp) were calculated by fitting the extended Tofts pharmacokinetic model to the DCE-MRI data, using the DCETool in Horos [27, 28]. Subsequently, mean T2 value and mean perfusion parameter values of the ROIs were calculated. The Tofts pharmacokinetic model is widely used for this purpose and has shown to be the most accurate model for patellar bone [29]. For highly vascularized tissues, like the IPFP, the extended Tofts pharmacokinetic model is more suitable due to the addition of the vascular term $\mathrm{Vp}$; therefore, in this study, we used the extended Tofts pharmacokinetic model [30]. Ktrans reflects the volume transfer constant into the tissue compartment, Kep describes the rate constant back into the vascular component, $\mathrm{Ve}$ is the extravascular extracellular space, and $\mathrm{Vp}$ is the vascular fraction of the region [31]. The arterial input function (AIF) was estimated using a ROI in the popliteal artery. All fitted AIFs were visually checked.

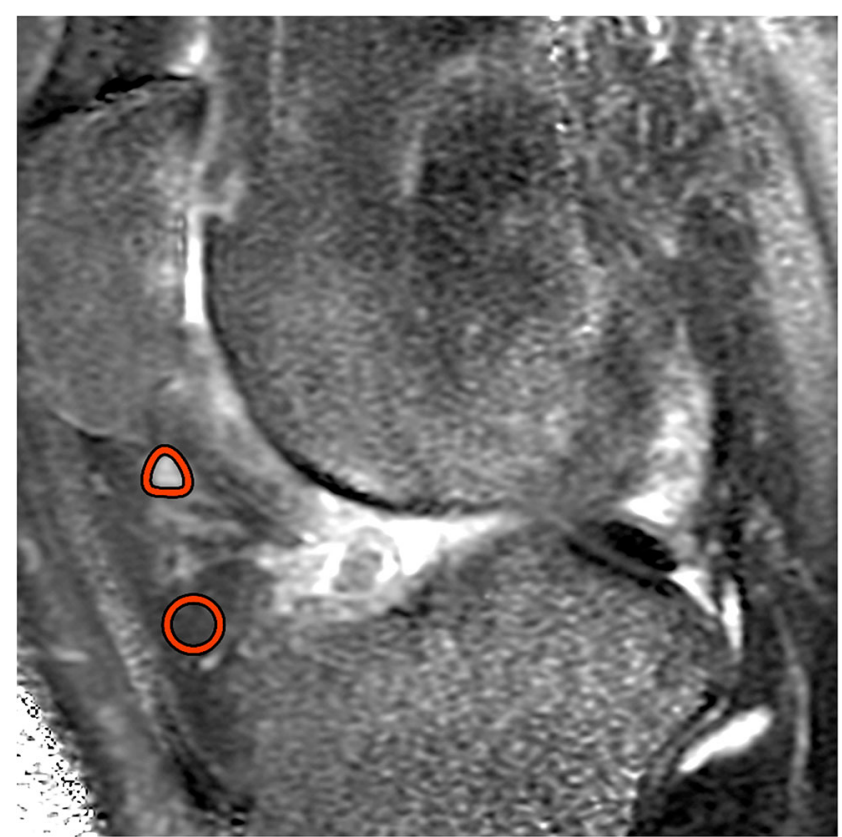

Fig. 1 Two ROIs were drawn in the IPFP, one within the $\mathrm{T}_{\mathrm{FS}^{-}}$ hyperintense region and the second in an adjacent area without T2hyperintensity

\section{Statistical analysis}

The image analyses result in a mean value for the $\mathrm{T} 2$ and perfusion parameters within each region. For each region, the median T2 and perfusion parameters over all subjects in a certain group were calculated, as well as the interquartile range (IQR) as a measure of variability. Since all DCE-MRI variables showed a non-normal distribution, using the Shapiro-Wilk test, a paired Wilcoxon signed-rank test was used to compare perfusion parameter values of the $\mathrm{T} 2_{\mathrm{FS}}-\mathrm{hy}-$ perintense region with the adjacent region with normal signal intensity within the different subject groups. A MannWhitney $U$ test was used to evaluate the location distribution of $\mathrm{T} 2 \mathrm{FS}_{\mathrm{F}}$-hyperintense IPFP regions over the groups as well as differences in DCE-MRI perfusion parameters of a central versus a peripheral $\mathrm{T} 2{ }_{\mathrm{FS}}$-hyperintense region. Statistical analysis was performed using SPSS v25 (IBM). $P$ values $<0.05$ were considered to be statistically significant.

\section{Results}

In total, 100 participants were included from both studies: 22 patients with knee OA, 35 patients with PFP, and 43 healthy controls. The mean BMI was higher in the OA group $\left(30.6 \mathrm{~kg} / \mathrm{m}^{2}\right)$ in comparison to the PFP and the control group with a mean BMI of 24.6 and $22.3 \mathrm{~kg} / \mathrm{m}^{2}$, respectively. The Knee Injury and Osteoarthritis Outcome Score (KOOS) indicated that pain symptoms were most severe in the OA group. Characteristics of all participants are shown in Table 1. 
Table 1 Characteristics of participants with $\mathrm{T} 2$ regions within IPFP

\begin{tabular}{lccll}
\hline $\begin{array}{l}\text { Groups } \\
\text { Parameter }\end{array}$ & OA Patients & PFP patients & Controls & Total \\
$N=16$ & $N=13$ & $N=14$ & $N=43$ \\
\hline Sex male (\%) & $5(31 \%)$ & $8(62 \%)$ & $7(50 \%)$ & $20(47 \%)$ \\
Age in years & $63.3 \pm 6.3^{\mathrm{a}}$ & $27.0 \pm 5.6^{\mathrm{a}}$ & $25.8 \pm 4.4^{\mathrm{a}}$ & $29.6^{\mathrm{b}}$ \\
& & & {$[24.0-60.0]$} \\
BMI in kg/m ${ }^{2}$ & $30.6 \pm 5.2^{\mathrm{a}}$ & $24.6 \pm 3.5^{\mathrm{a}}$ & $22.3 \pm 2.2^{\mathrm{a}}$ & $24.3^{\mathrm{b}}$ \\
& & & $100.0^{\mathrm{b}}$ & {$[21.9-29.1]$} \\
KOOS pain subscale & $40.5 \pm 11.0^{\mathrm{a}}$ & $71.6 \pm 17.9^{\mathrm{a}}$ & {$[100.0-100.0]$} & $66.7^{\mathrm{b}}$ \\
& & & & {$[44.4-100.0]$} \\
\hline
\end{tabular}

$S D$ : standard deviation, $I Q R$ : interquartile range

${ }^{\mathrm{a}}$ Mean $\pm \mathrm{SD}$

${ }^{\mathrm{b}}$ Median [IQR]

$\mathrm{T} 2_{\mathrm{FS}}$-hyperintense IPFP regions were present in 43 subjects. The prevalence of the $\mathrm{T} 22_{\mathrm{FS}}$-hyperintense IPFP regions was different between the groups: 16 of $22(73 \%)$ knee OA patients, 13 of 35 (37\%) PFP patients, and 14 of 43 (33\%) controls. Of the 16 knee OA patients, three had a radiographic OA severity of Kellgren and Lawrence grade 2, eight had KL grade 3, and five patients had KL grade 4 .

The median T2 value in IPFP tissue without T2hyperintensity was $36.4,33.9$, and $32.7 \mathrm{~ms}$ in OA patients, PFP patients, and controls, respectively. For the $\mathrm{T} 2_{\mathrm{FS}}$-hyperintense regions, these values were $61.4,52.3$, and $53.7 \mathrm{~ms}$, respectively (Table 2).

Most hyperintense regions were located centrally $(n=30)$ in the IPFP whereas 13 were located more peripherally. We observed no significant difference in location distribution between groups as well as no difference in all DCE-MRI perfusion parameters of a central versus a peripheral $\mathrm{T} 2_{\mathrm{FS}}$ hyperintense region.

In knee OA patients, the $\mathrm{T} 2{ }_{\mathrm{Fs}}$-hyperintense IPFP regions demonstrated significantly higher values of Ktrans (see Fig. 2) and Ve compared to tissue with normal signal intensity (0.039 $\mathrm{min}^{-1}$ vs. $0.025 \mathrm{~min}^{-1}$ for Ktrans and 0.157 vs. 0.119 for $\mathrm{Ve}$ ). Kep and $\mathrm{Vp}$ were higher within $\mathrm{T} 2_{\mathrm{FS}}$-hyperintense lesions compared to tissue with normal signal intensity (median Kep 197.57 vs. 163.49 and median Vp 2.09 vs. 1.03 , respectively). However, these differences were not statistically significant for both Kep $(p=0.079)$ and $\operatorname{Vp}(p=0.363)$. In both controls and PFP patients, all DCE-MRI perfusion

Table 2 T2 and DCE-MRI perfusion parameters in the IPFP. $p$ values of the difference between T2-hyperintense region and tissue with normal signal intensity are reported. $I Q R=$ interquartile range

\begin{tabular}{|c|c|c|c|c|c|c|c|c|c|c|c|c|c|c|}
\hline & \multicolumn{2}{|c|}{$\begin{array}{l}\mathrm{T} 2 \text { relaxation } \\
\text { time }(\mathrm{ms})\end{array}$} & \multicolumn{3}{|c|}{ Ktrans $\times 1000\left(\mathrm{~min}^{-1}\right)$} & \multicolumn{3}{|c|}{$\operatorname{Kep} \times 1000\left(\mathrm{~min}^{-1}\right)$} & \multicolumn{3}{|c|}{$\mathrm{Ve} \times 1000$ (unit-less) } & \multicolumn{3}{|c|}{$\mathrm{Vp} \times 1000$ (unit-less) } \\
\hline & Median & IQR & Median & $\mathrm{IQR}$ & $\begin{array}{l}p \\
\text { value }^{\dagger}\end{array}$ & Median & IQR & $\begin{array}{l}p \\
\text { value }^{\dagger}\end{array}$ & Median & IQR & $\begin{array}{l}p \\
\text { value }^{\dagger}\end{array}$ & Median & IQR & $\begin{array}{l}p \\
\text { value }^{\dagger}\end{array}$ \\
\hline \multicolumn{15}{|l|}{ OA patients } \\
\hline $\begin{array}{l}\text { T2-hyperintense } \\
\text { region }\end{array}$ & 61.40 & 31.27 & 39.03 & 65.79 & $0.017 *$ & 197.57 & 198.66 & 0.079 & 157.19 & 259.45 & $0.010 *$ & 2.09 & 6.15 & 0.363 \\
\hline $\begin{array}{l}\text { Normal signal } \\
\text { intensity }\end{array}$ & 36.39 & 6.15 & 24.73 & 22.76 & & 163.49 & 131.83 & & 119.18 & 151.43 & & 1.03 & 5.98 & \\
\hline \multicolumn{15}{|l|}{ PFP patients } \\
\hline $\begin{array}{l}\text { T2-hyperintense } \\
\text { region }\end{array}$ & 52.30 & 10.66 & 11.07 & 13.49 & 0.552 & 173.41 & 198.61 & 0.552 & 143.00 & 128.37 & 0.917 & 0.22 & 0.52 & 0.477 \\
\hline $\begin{array}{l}\text { Normal signal } \\
\text { intensity }\end{array}$ & 33.85 & 4.62 & 13.61 & 10.09 & & 112.86 & 181.96 & & 143.95 & 125.33 & & 0.11 & 0.39 & \\
\hline \multicolumn{15}{|l|}{ Controls } \\
\hline $\begin{array}{l}\text { T2-hyperintense } \\
\text { region }\end{array}$ & 53.67 & 15.49 & 9.84 & 17.28 & 0.363 & 91.00 & 97.38 & 0.778 & 160.62 & 255.09 & 0.510 & 0.13 & 0.70 & 0.075 \\
\hline $\begin{array}{l}\text { Normal signal } \\
\text { intensity }\end{array}$ & 32.71 & 4.74 & 14.36 & 23.02 & & 122.28 & 117.59 & & 181.53 & 116.12 & & 0.01 & 0.18 & \\
\hline
\end{tabular}

${ }^{\dagger}$ Wilcoxon signed-rank test

$* p$ values $<0.05$ 


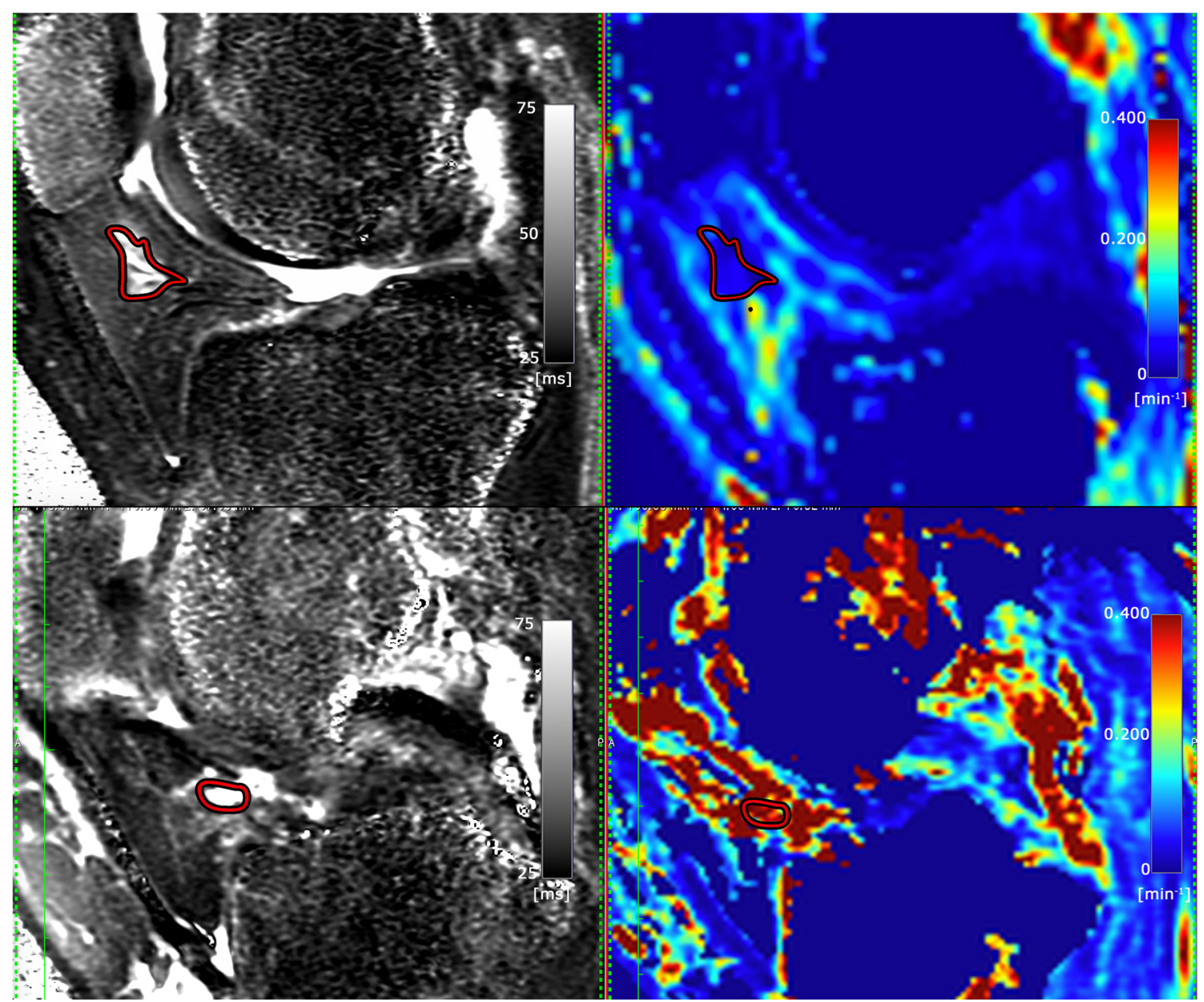

Fig. 2 Delineated T2-hyperintense region within IPFP on T2 map (left) and corresponding Ktrans map (values in 1/min) (right) in patient with PFP (upper row) and patient with OA (lower row). Higher values of Ktrans are depicted in red

parameters were not significantly different between IPFP tissue with and without a $2_{\mathrm{FS}}$-hyperintensity. In PFP-patients, a Ktrans of $0.014 \mathrm{~min}^{-1}$ and Kep $0.113 \mathrm{~min}^{-1}$ in IPFP tissue with normal signal intensity and a Ktrans of $0.011 \mathrm{~min}^{-1}$ and Kep $0.173 \mathrm{~min}^{-1}$ in tissue with $\mathrm{T} 2 \mathrm{FS}$-hyperintensity was found. In controls, the median Ktrans was $0.014 \mathrm{~min}^{-1}$ and median Kep was $0.122 \mathrm{~min}^{-1}$ in IPFP tissue with normal signal intensity and in tissue with $\mathrm{T} 2{ }_{\mathrm{FS}}$-hyperintensity these values were 0.010 and $0.091 \mathrm{~min}^{-1}$, respectively. Moreover, all DCE-MRI perfusion parameters were higher in both the hyperintense lesions and normal IPFP tissue in the OA group. All DCE-MRI results are shown in Table 2.

\section{Discussion}

In this study, quantitative DCE-MRI perfusion parameters were measured within $\mathrm{T} 2{ }_{\mathrm{FS}}$-hyperintense regions and adjacent IPFP tissue with normal signal intensity of patients with knee $\mathrm{OA}$ and patients with PFP and in healthy controls. Our hypothesis was that identically appearing $\mathrm{T} 2 \mathrm{FS}_{\mathrm{FS}}$-hyperintense
IPFP regions in patients with OA, PFP, and control subjects demonstrate different degrees of increased perfusion measured with quantitative DCE-MRI compared to adjacent IPFP tissue with normal signal intensity. We expected the highest perfusion in patients with OA, in which term Hoffa synovitis has been coined to describe such regions. Indeed, we found that $\mathrm{T} 2{ }_{\mathrm{FS}}$-hyperintense regions showed significantly increased perfusion compared to adjacent IPFP tissue with normal signal intensity in OA patients only, in contrast to both patients with PFP and healthy controls. This finding suggests an inflammatory pathogenesis of such regions in OA patients, but not in patients with PFP and healthy control subjects. Our observation that knee OA patients demonstrated, in general, higher DCE-MRI perfusion parameters than PFP patients and healthy controls, irrespective of the presence of a $\mathrm{T}_{\mathrm{FS}}$-hyperintense region, also indicate that the entire IPFP may be affected by inflammation in OA and possibly also by neo-angiogenesis, based on the elevated $\mathrm{Vp}$, which represents the vascular fraction within the ROI. Our observation of this phenomenon in the IPFP is of interest, as from previous literature it is known that $\mathrm{OA}$ is not a simple "wear and tear" disease of 
cartilage and bone, but a whole organ disease, including several soft tissues such as the IPFP [32]. Accordingly, there is an increasing focus on systemic treatment approaches for OA, such as anti-inflammatory and anti-angiogenic medication [33]. In future trials, it will be a prerequisite to identify OA subtypes, in which advanced MR imaging, such as DCEMRI, could potentially play a major role.

The different results for PFP found in this study are not consistent with current insights in PFP, which is supposed to be a precursor of OA $[34,35]$. A possible explanation might be that tissue homeostasis is not yet as disturbed in PFP and inflammatory cytokines are not yet released. Thus, even though T2 hyperintense IPFP regions appear identically on unenhanced T2-weighted fat-saturated MR images in OA and PFP patients as well as healthy controls, the results of our DCE-MRI analysis show that there are different degrees of perfusion within the IPFP of controls, PFP patients, and OA patients, which may point towards different pathophysiologies. This knowledge will help the practicing radiologist who is confronted with an increased application of sensitive knee MRI to appraise these lesions in the context of the patient's age and concurrent abnormalities.

In this study, the IPFP was quantitatively analyzed by $\mathrm{T} 2$ mapping and DCE-MRI in order to investigate the pathophysiology of T2 signal alterations in the IPFP within OA and PFP. The single prior study that applied DCE-MRI to investigate the IPFP by Ballegaard et al [23] used a different approach in the definition of the region of interest, as they focused on the entire IPFP in 3D rather than $\mathrm{T} 2{ }_{\mathrm{FS}}$-hyperintensities within the fat pad. Furthermore, only obese patients with knee OA were included and a heuristic DCE-MRI analysis method without pharmacokinetic modeling was performed.

A strength of the current study is the quantitative assessment of DCE-MRI perfusion values, which offers more robust parameters that directly represent the microvasculature physiology, in contrast to semi-quantitative analysis. Furthermore, the inclusion of different patient groups from two studies offered the possibility to determine the nature of $\mathrm{T} 2_{\mathrm{FS}}$-hyperintense IPFP regions across different disease entities, one of which (PFP) has been suggested as a precursor to the each other $(\mathrm{OA})$. We were able to directly compare the results of the quantitative DCE-MRI analysis from both studies because the exact same MRI scanner was used with identical scan and image post processing for both studies. Additionally, statistical analyses were performed within subjects of each subgroup, and thus possible differences in confounding variables between the subgroups will not have influenced our results.

A potential limitation was that no $\mathrm{B} 1+$ inhomogeneity assessment and $\mathrm{T} 1$ correction was possible, due to the lack of $\mathrm{B} 1+$ or pre-contrast T1 map. A fixed T1(0) value of $1443 \mathrm{~ms}$ (standard value of the DCE Tool used in Horos) was used instead. We expect that differences that may have arisen as a result of ignoring region $\mathrm{T} 1$ variability will not change the outcome of this study, as the observed differences in perfusion were large and substantially larger than any differences that we would expect due to T1 variability. Furthermore, we used a dedicated transmit/receive knee coil with relatively homogeneous B1 field. At the time of the MR acquisitions, linear gadolinium contrast agents, like gadopentetate dimeglumine, were commonly in use. Since then, these have been withdrawn from the EU market and have been replaced by alternatives that carry less risk for nephrogenic systemic fibrosis. As the perfusion kinetics of these alternatives are similar, we expect our results to be relevant for the newer generation contrast agents as well. Another limitation is that the OA group comprised patients referred for knee arthroplasty because of end-stage clinical OA, although the radiographic OA severity ranged from KL grade 2 to 4 , with grade 4 relatively underrepresented. Furthermore, ROIs were drawn on one slice only. Finally, $\mathrm{T}_{\mathrm{FS}}$-hyperintense lesions were found only in 43 subjects, and the OA group was relatively small. However, all these subjects underwent an extensive MRI protocol including the administration of an intravenous contrast agent. In future research, it would be interesting to examine the perfusion of $\mathrm{T} 2_{\mathrm{FS}}$ hyperintense lesions in a population with a wider range of clinical OA severity, to evaluate the diagnostic value of $\mathrm{T} 2 \mathrm{FS}_{\mathrm{FS}}$-hyperintense lesions and their perfusion characteristics in classifying patients with unknown OA status, and to study the relationship of perfusion parameters with clinical symptoms.

In conclusion, $\mathrm{T} 2 \mathrm{FS}_{\mathrm{FS}}$-hyperintense regions of the IPFP demonstrated higher quantitative DCE-MRI blood perfusion parameters compared to adjacent tissue with normal signal intensity in patients with knee OA, but not in patients with PFP and healthy control subjects. This suggests different pathophysiology of IPFP $\mathrm{T} 2_{\mathrm{FS}}$-hyperintense regions across patient subgroups, in which an inflammatory pathogenesis is only present in OA.

Acknowledgments We would like to acknowledge Kyung Hyun Sung for the development of the DCE tool in Horos.

Funding information This research was partially funded by a Young Researchers Grant awarded by the European Society of Musculoskeletal Radiology, a seed grant from the Radiological Society of North America (RSNA), and a EUR fellowship from the Erasmus University Rotterdam and the Dutch Arthritis Foundation.

\section{Compliance with ethical standards}

Guarantor The scientific guarantor of this publication is Edwin H.G. Oei.

Conflict of interest The authors of this manuscript declare no relationships with any companies, whose products or services may be related to the subject matter of the article.

Statistics and biometry No complex statistical methods were necessary for this paper. 
Informed consent Written informed consent was obtained from all subjects (patients) in this study.

Ethical approval Institutional Review Board approval was obtained.

Study subjects or cohorts overlap Some study subjects or cohorts have been previously reported in 2 articles:

van Tiel J, Kotek G, Reijman M, et al (2016) Is T1 $\rho$ Mapping an Alternative to Delayed Gadolinium-enhanced MR Imaging of Cartilage in the Assessment of Sulphated Glycosaminoglycan Content in Human Osteoarthritic Knees? An in Vivo Validation Study. Radiology 279:523531. https://doi.org/10.1148/radiol.2015150693

van der Heijden RA, de Kanter JL, Bierma-Zeinstra SM, et al (2016) Structural Abnormalities on Magnetic Resonance Imaging in Patients With Patellofemoral Pain: A Cross-sectional Case-Control Study. Am J Sport Med 44:2339-2346. https://doi.org/10.1177/0363546516646107

\section{Methodology}

- Retrospective analysis of two prospective studies

- diagnostic study

- performed at one institution

Open Access This article is licensed under a Creative Commons Attribution 4.0 International License, which permits use, sharing, adaptation, distribution and reproduction in any medium or format, as long as you give appropriate credit to the original author(s) and the source, provide a link to the Creative Commons licence, and indicate if changes were made. The images or other third party material in this article are included in the article's Creative Commons licence, unless indicated otherwise in a credit line to the material. If material is not included in the article's Creative Commons licence and your intended use is not permitted by statutory regulation or exceeds the permitted use, you will need to obtain permission directly from the copyright holder. To view a copy of this licence, visit http://creativecommons.org/licenses/by/4.0/.

\section{References}

1. Draghi F, Ferrozzi G, Urciuoli L, Bortolotto C, Bianchi S (2016) Hoffa's fat pad abnormalities, knee pain and magnetic resonance imaging in daily practice. Insights Imaging 7:373-383

2. Felson DT (2005) The sources of pain in knee osteoarthritis. Curr Opin Rheumatol 17:624-628

3. Dye SF (2005) The pathophysiology of patellofemoral pain: a tissue homeostasis perspective. Clin Orthop Relat Res 436:100-110

4. Clockaerts S, Bastiaansen-Jenniskens YM, Runhaar J et al (2010) The infrapatellar fat pad should be considered as an active osteoarthritic joint tissue: a narrative review. Osteoarthritis Cartilage 18: 876-882

5. Biedert RM, Sanchis-Alfonso V (2002) Sources of anterior knee pain. Clin Sports Med 21(335-47):vii

6. Ioan-Facsinay A, Kloppenburg M (2013) An emerging player in knee osteoarthritis: the infrapatellar fat pad. Arthritis Res Ther 15: 225. https://doi.org/10.1186/ar4422

7. Hunter DJ, Guermazi A, Lo GH et al (2011) Evolution of semiquantitative whole joint assessment of knee OA: MOAKS (MRI Osteoarthritis Knee Score). Osteoarthritis Cartilage 19:990-1002

8. Atukorala I, Kwoh CK, Guermazi A et al (2016) Synovitis in knee osteoarthritis: a precursor of disease? Ann Rheum Dis 75:390-395

9. Han W, Aitken D, Zhu Z et al (2016) Signal intensity alteration in the infrapatellar fat pad at baseline for the prediction of knee symptoms and structure in older adults: a cohort study. Ann Rheum Dis 75:1783-1788
10. Ruhdorfer A, Haniel F, Petersohn T et al (2017) Between-group differences in infra-patellar fat pad size and signal in symptomatic and radiographic progression of knee osteoarthritis vs non-progressive controls and healthy knees - data from the FNIH Biomarkers Consortium Study and the Osteoarthritis In. Osteoarthritis Cartilage 25:1114-1121

11. Wang K, Ding C, Hannon MJ et al (2018) Signal intensity alteration within infrapatellar fat pad predicts knee replacement within 5 years: data from the osteoarthritis initiative. Osteoarthritis Cartilage 26:1345-1350

12. Wang K, Ding C, Hannon MJ, Chen Z, Kwoh CK, Hunter DJ (2019) Quantitative signal intensity alteration in Infrapatellar fat pad predicts incident radiographic osteoarthritis: the osteoarthritis initiative. Arthritis Care Res (Hoboken) 71:30-38

13. van der Heijden RA, de Kanter JL, Bierma-Zeinstra SM et al (2016) Structural abnormalities on magnetic resonance imaging in patients with patellofemoral pain: a cross-sectional case-control study. Am J Sports Med 44:2339-2346. https://doi.org/10.1177/ 0363546516646107

14. Roemer FW, Guermazi A, Zhang Y et al (2009) Hoffa's fat pad: evaluation on unenhanced MR images as a measure of patellofemoral synovitis in osteoarthritis. AJR AJR Am J Roentgenol 192:1696-1700

15. Subhawong TK, Eng J, Carrino JA, Chhabra A (2010) Superolateral Hoffa's fat pad edema: association with patellofemoral maltracking and impingement. AJR Am J Roentgenol 195:1367-1373

16. Link TM, Li X (2011) Bone marrow changes in osteoarthritis. Semin Musculoskelet Radiol 15:238-246

17. Sourbron SP, Buckley DL (2012) Tracer kinetic modelling in MRI: estimating perfusion and capillary permeability. Phys Med Biol 57: $1-33$

18. Boesen M, Kubassova O, Bouert R et al (2012) Correlation between computer-aided dynamic gadolinium-enhanced MRI assessment of inflammation and semi-quantitative synovitis and bone marrow oedema scores of the wrist in patients with rheumatoid arthritis-a cohort study. Rheumatology (Oxford) 51:134-143

19. Axelsen MB, Stoltenberg M, Poggenborg RP et al (2012) Dynamic gadolinium-enhanced magnetic resonance imaging allows accurate assessment of the synovial inflammatory activity in rheumatoid arthritis knee joints: a comparison with synovial histology. Scand J Rheumatol 41:89-94

20. Boesen M, Kubassova O, Sudol-Szopinska I et al (2018) MR imaging of joint infection and inflammation with emphasis on dynamic contrast-enhanced MR imaging. PET Clin 13:523-550

21. Riis RG, Gudbergsen H, Henriksen M et al (2016) Synovitis assessed on static and dynamic contrast-enhanced magnetic resonance imaging and its association with pain in knee osteoarthritis: a cross-sectional study. Eur J Radiol 85:1099-1108

22. Riis RG, Gudbergsen H, Simonsen $\mathrm{O}$ et al (2017) The association between histological, macroscopic and magnetic resonance imaging assessed synovitis in end-stage knee osteoarthritis: a crosssectional study. Osteoarthritis Cartilage 25:272-280

23. Ballegaard C, Riis RG, Bliddal $\mathrm{H}$ et al (2014) Knee pain and inflammation in the infrapatellar fat pad estimated by conventional and dynamic contrast-enhanced magnetic resonance imaging in obese patients with osteoarthritis: a cross-sectional study. Osteoarthritis Cartilage 22:933-940

24. Kellgren JH, Lawrence JS (1957) Radiological assessment of osteoarthrosis. Ann Rheum Dis 16:485-493

25. van Tiel J, Kotek G, Reijman $M$ et al (2016) Is T1 $\rho$ mapping an alternative to delayed gadolinium-enhanced MR imaging of cartilage in the assessment of sulphated glycosaminoglycan content in human osteoarthritic knees? An in vivo validation study. Radiology 279:523-531. https://doi.org/10.1148/radiol.2015150693 
26. Klein S, Staring M, Murphy K, Viergever MA, Pluim J (2010) Elastix: a toolbox for intensity-based medical image registration. IEEE Trans Med Imaging 29:196-205

27. Tofts PS, Kermode AG (1991) Measurement of the blood-brain barrier permeability and leakage space using dynamic MR imaging. 1. Fundamental concepts. Magn Reson Med 17:357-367

28. Sung K (2015) DCE tool. Body Magnetic Resonance Group Stanford University, Stanford. Available via http://kyungs.bol. ucla.edu/software/DCE_tool/DCE_tool.html. Accessed 23 May 2018

29. Poot DHJ, van der Heijden RA, van Middelkoop M, Oei EHG, Klein S (2018) Dynamic contrast-enhanced MRI of the patellar bone: how to quantify perfusion. J Magn Reson Imaging 47:848858

30. Sourbron SP, Buckley DL (2011) On the scope and interpretation of the Tofts models for DCE-MRI. Magn Reson Med 66:735-745

31. Tofts PS, Brix G, Buckley DL et al (1999) Estimating kinetic parameters from dynamic contrast-enhanced T(1)-weighted MRI of a diffusable tracer: standardized quantities and symbols. J Magn Reson Imaging 10:223-232

32. Loeser RF, Goldring SR, Scanzello CR, Goldring MB (2012) Osteoarthritis: a disease of the joint as an organ. Arthritis Rheum 64:1697-1707

33. Ghouri A, Conaghan PG (2019) Update on novel pharmacological therapies for osteoarthritis. Ther Adv Musculoskelet Dis 11:1-11

34. Thomas MJ, Wood L, Selfe J, Peat G (2010) Anterior knee pain in younger adults as a precursor to subsequent patellofemoral osteoarthritis: a systematic review. BMC Musculoskelet Disord 11. https://doi.org/10.1186/1471-2474-11-201

35. Eijkenboom JFA, Waarsing JH, Oei EHG, Bierma-Zeinstra SMA, van Middelkoop M (2018) Is patellofemoral pain a precursor to osteoarthritis? Bone Joint Res 7:541-547

Publisher's note Springer Nature remains neutral with regard to jurisdictional claims in published maps and institutional affiliations. 